\title{
Policy Frames sobre Reinserción Social de varones privados de libertad en Chile durante el período 2012-2013.
}

Análisis de los conflictos y convergencias en los discursos de sus actores clave.

\author{
Jorge Maluenda \\ Universidad de Concepción, Chile
}

\begin{abstract}
Resumen
La presente investigación analiza los Policy Frame utilizados por los principales actores involucrados en Reinserción Social de adultos varones privados de libertad en Chile, en el período 2012-2013. Se identifican los frames dominantes y aquellos en conflicto. Para llevar a cabo este análisis se han utilizado dos técnicas metodológicas: la Grounded Theory (Strauss y Corbin, 2002) y el Policy Frame Analysis. A partir de los distintos Frames observados en los actores estudiados, se ha podido establecer la presencia de ejes comunes, complementarios y fuentes de posible conflicto, además de espacios para la convergencia en la relación de actores ante diversos ejes.
\end{abstract}

Palabras clave: Reinserción social, Policy Frame Analysis, Grounded Theory, delincuencia

Social Reinsertion Frame Policys of male prisoners in Chile: 2012-2013. Analysis of conflicts and convergences in the speeches of their key actors

\begin{abstract}
This research analyses the Policy Frame used by the leading actors involved in a social reintegration process of adult male prisoners in Chile, during the 2012-2013 period; identifying dominant frames and those in conflict. For this analysis we have been used two methodological techniques: Grounded Theory (Strauss and Corbin, 2002) and the Policy Frame Analysis. From the various frames observed in the studied actors, it has been able to establish the presence of common axes, complementary axes and sources of potential conflict was concluded; also was the presence of common aspects between different axes and actors.
\end{abstract}

Keywords: Social reinsertion, Policy Frame Analysis, Grounded Theory, delinquency

\section{Policy frames sobre reinserción social de varones privados de libertad en Chile durante el período 2012-2013}

En Chile, la seguridad pública ha sido una de las principales preocupaciones de la ciudadanía durante los últimos 20 años. El Gobierno de Chile, en su afán por mejorar las condiciones de seguridad ciudadana, ha decidido adoptar distintas estrategias que le permitan abordar de manera integral el fenómeno de la delincuencia, siendo uno de sus hitos clave, el lanzamiento de la Estrategia Nacio-

\footnotetext{
*Dirección de correspondencia [Correspondence address]:

Jorge Maluenda, Universidad de Concepción, Chile

E-mail: jorgemaluenda@udec.cl
}

nal de Seguridad Pública (2006-2010). Es a través de este instrumento, que se reformula la prioridad de los objetivos de la política gubernamental, donde la Rehabilitación y Reinserción Social adquiere una mayor importancia a nivel estratégico (Pantoja et al., 2009). Esta última ha sido un tema de especial interés, sobre todo en su calidad de intervención frente al ciclo de la delincuencia, ya que se le considera "una eficiente forma de prevenir el delito, mediante la atención y asistencia del penado" (Pantoja et al., 2009), interesando a distintos actores preocupados por el problema de la delincuencia, quienes se involucran en el debate a través de la discusión académica respecto de las mejores formas para intervenir.

Es en este escenario que distintos actores intentan plasmar sus perspectivas, generando un debate respecto de cómo debiese ser la política pública de Reinserción Social de personas privadas de libertad, como parte integral de la respuesta pública frente 
al problema de la seguridad ciudadana.

Por ello, a través de la presente investigación, se busca analizar los policy frames que impulsan a los principales actores involucrados en Reinserción Social de adultos varones privados de libertad en Chile, en el período 2012-2013. Lo anterior implica identificar los principales actores, caracterizar los policy frames presentes en sus prácticas y discursos, así como relacionarlos con las prácticas y discursos de otros actores involucrados. Con esto, no solo se espera aportar con conocimiento respecto al panorama general que exhibe Chile para la mejor construcción de la política pública en esta materia, sino que también se busca aportar con sugerencias que permitan abordar de manera más eficiente y coherente este complejo e irresoluto tema.

\section{El Policy Frames Analysis}

Esta investigación se centra en las primeras tres etapas del ciclo de políticas públicas (Roth, 2002; Jones, 1970), esto es: identificación del problema, formulación de decisiones y toma de decisiones. Estas etapas conforman el proceso de diseño de la política pública, a través del cual se la va moldeando y definiendo (Santibañez et al., 2013), a través de la definición de los problemas y sus definición, siendo el trasfondo o espíritu que da sentido a la toma de decisiones (Jones, 1970).

De esta manera, se comprenden las políticas públicas como el tránsito desde la diversidad de ideas y problemáticas observadas por la ciudadanía hacia la agenda social y luego hacia la agenda política, siendo aquí donde los temas pueden o no transformarse en programas, es decir, acciones concretas del poder estatal (Lahera, 2002).

Notamos entonces que el proceso de Formulación, implica un juego dinámico entre actores que interactúan en distintos escenarios. Para Hidalgo (2001), en el abordaje de la política pública deben estudiarse los actores en juego, puesto que estos, interactúan y tratan de hacer prevalecer sus intereses. Meltsner (1992), por su parte, afirma que los distintos actores partícipes de una política se reconocen por sus posturas de aceptación o rechazo hacia esta, desplegando una gama de recursos para ello.

De esta forma, las distintas construcciones sobre el problema público, son producto de la negociación entre diversos actores relevantes en determinados escenarios, con mayor o menor grado de injerencia sobre los diversos temas que se abordan (Subirats, 2008; Banco Interamericano De Desarrollo, 2006). Más aún, como señala Martin (2012), el proceso de construcción de los marcos interpretativos de la política, responde a un diálogo y negociación no solo de actores, sino que también entre distintas perspectivas y visiones sobre el problema.

Estas formas de comprensión del problema, implican perspectivas respecto de cómo delimitarlo, cuáles son sus causas y los posibles cursos de acción para enfrentarlos, las que pueden ser comprendidas dentro de distintos marcos interpretativos sobre la política - Policy Frame -, un principio de organización que transforma la información fragmentada o casual en un problema político estructurado (Verloo y van Lamoen, 2002).

A través de estos, los distintos actores "enmarcan" la realidad empírica que se les presenta, interpretando los problemas sociales, más que como fenómenos empíricos, como situaciones construidas y legitimadas por dichos actores mediante el lenguaje (Cuvardic, 2001). Goffman (2006) señala que el marco interpretativo (Frame) para organizar la experiencia se denominará marco de trabajo, el cual "permite a su usuario localizar percibir, identificar y etiquetar en sus términos un número aparentemente infinito de situaciones concretas". Esto quiere decir, que el Frame permite al individuo definir una determinada problemática discriminando aquellos elementos que tienen sentido de aquellos que son tangenciales o distintos.

El análisis de los marcos interpretativos (Policy frame analysis), como lo señalan Bustelo y Lombardo $(2005 ; 2006)$, se concibe como el estudio de los marcos interpretativos de la política (Policy Frame) y cómo estos se encuentran involucrados en la construcción y negociación de la realidad por parte de los actores sociales y políticos en sus respectivos discursos. Por lo tanto, esta herramienta busca descifrar el mapa de relaciones entre los policy frames a la base de los discursos de distintos actores sobre un tema político en específico.

Para Fischer (2003) el análisis de los Frames, se entiende como el análisis y reestructuración de las posiciones y discursos de los actores sociales y políticos. Estos negocian sobre la identificación y alternativas de solución de un problema. Este proceso considera las estructuras de creencias, apreciaciones y percepciones de quienes se encuentran involucrados (Fischer, 2003).

Así, según lo sugieren Schön y Rein (1995), en el policy frame analysis se identifican los frames dominantes y aquellos en conflicto entre sí y que se constituyen para dar sentido a diferentes situaciones, atribuir culpas o causalidades y sugerir líneas de actuación. Se puede hablar, por lo tanto de Frames hegemónicos, complementarios y antagonistas (Martin, 2012). Los frames hegemónicos, son aque- 
llos compartidos por la mayoría de los actores; los frames complementarios, no son mayoritarios, pero no contradicen las ideas compartidas por los frames hegemónicos; por último, los frames antagonistas, refieren a posturas minoritarias que están en oposición a las ideas de los frames hegemónicos.

De esta forma, es posible observar los frames y las relaciones establecidas entre ellos, en el sentido de contraposiciones, conflictos, convergencias, dominio y complementariedad, a partir de los discursos emitidos por los principales actores involucrados en la materia.

\section{Policy Frames en el abordaje de la delincuencia}

A partir de Morales (2012) es posible identificar la presencia de diversos frames presentes en la historia de la política pública para abordar la criminalidad en Chile. Reconocemos, de esta forma, enfoques de carácter positivista (Enfoque de tratamiento y rehabilitación a comienzos del siglo $\mathrm{XX}$ ), aquellos que centran las causas en el contexto social (1920-1930), además del auge de la tesis de la defensa social (1930). Posteriormente, hay un período de letargo en la segunda mitad del siglo $\mathrm{XX}$, así como en el período de dictadura militar. Este último, es producto del enfoque sobre el llamado "enemigo interno" (Morales, 2012), dejando en segundo plano la delincuencia común como tema central de la política de seguridad ciudadana. En los 90‘s, en un tránsito hacia la democracia, la tarea se transforma en moderar el poder y alcance de las Fuerzas Armadas, debiendo dialogar y negociar extensamente en temas de Derechos Humanos e incorporando paulatinamente la exigencias de la política internacional para el tratamiento de la criminalidad (Morales Peillard, 2012).

Sin embargo, se ha observado a fines de los 90 y comienzos de los 2000, la aparición paulatina de una serie de estrategias que responden a intentos de abordar la delincuencia en su complejidad (sistémica) lo que abre el espacio a la discusión sobre la Rehabilitación de privados de libertad con mayor fuerza que lo observado previamente. Más aún, este espacio en la discusión, así como los recursos destinados a la intervención y sistematización de experiencias en distintos ámbitos, abren la posibilidad de plantear con fundamentos - investigación basada en evidencia - y alternativas de trabajo como la Reinserción Social.

En relación a lo anterior, Uprimny y Saffon (2006) plantean que la política en el ámbito penal, acorde a los tiempos y la realidad internacional, ha venido evolucionando, ajustándose a un paradigma restaurativo, entendido como aquel al alternativo y crítico del funcionamiento del sistema penal, en relación a la modalidad de castigo frente a las formas ordinarias del crimen presentes en la sociedad, y que se enmarca en un movimiento más amplio, de crítica al carácter represivo y retributivo del derecho penal (Uprimny y Saffon, 2006). En la misma línea, Escobar (2008) plantea que este paradigma comienza a incorporar el concepto de derechos de víctimas y victimarios, y con ello opciones de rehabilitación para los segundos.

En una perspectiva actual, con intención de integración, Andrews y Bonta (2010), proponen el modelo Riesgo-Necesidad-Responsividad (RNR), dirigido principalmente al tratamiento de la delincuencia a través de estos tres grande principios, uno de los modelos principales a nivel internacional, a la vez que importante influencia en los modelos y programas del Ministerio del Interior y Seguridad Pública.

El principio de Riesgo sugiere que la reincidencia puede ser reducida si el nivel de las prestaciones entregadas al infractor es proporcional al riesgo de reincidencia del individuo; el de Necesidad plantea que la intervención debe centrarse en las necesidades criminógenas, es decir aquellos factores de riesgo dinámico que están vinculados directamente con el comportamiento infractor, agregando que los esfuerzos no deben enfocarse sobre los factores de riesgo estáticos, puesto que estos solo cambian en una dirección (aumento) y son inmutables ante el tratamiento. El principio de Responsividad (disposición a responder), alude a que las intervenciones de aprendizaje social son las formas más efectivas para enseñar nuevos comportamientos a personas, y que, para generar la direccionalidad intencionada del cambio, estas deben encontrarse ajustadas a la realidad concreta del individuo en cuestión (Pantoja et al., 2009).

Estos distintos enfoques para la comprensión del problema, impulsan la discusión respecto del rol del estado y la familia, cuestionando el tradicional abordaje del delito como una disposición estable donde la responsabilidad del delito recae directamente en el involucrado.

\section{Reinserción social en Chile}

Con respecto a la Reinserción Social en Chile, tal como señala Lagos (2012), el recorrido de las políticas rectoras respecto del tratamiento de estas temáticas ha transitado desde la aparición de la Política Nacional de Seguridad Ciudadana (20032004), pasando por la Estrategia Nacional de Seguridad Pública (2006-2010), y llegando a la ac- 
tualidad hasta el Plan de Seguridad Pública Chile Seguro (2010-2014).

La Estrategia Nacional de Seguridad Pública, trae consigo la instalación de 6 ejes fundamentales para el trabajo sobre la delincuencia en nuestro país: fortalecimiento institucional, información, prevención, control y sanción, rehabilitación, y la asistencia judicial y social de víctimas. Uno de los aspectos interesantes a destacar en el foco de esta estrategia es su "carácter intersectorial" (Lagos, 2012), razón por la cual, se explicita la participación de algunos actores gubernamentales fundamentales para el funcionamiento de la misma (Ministerio de Justicia de Chile, 2013).

Por su parte, la política "Plan de Seguridad Pública Chile Seguro", actualiza los ejes principales de trabajo incorporados en las ENSP, dando continuidad al trabajo comenzado en 2006, pero incorporando nuevos elementos que cambian la focalización de esta política pública. Así, ésta política, según Lagos (2012), recalca el carácter desconcentrado de las políticas de seguridad, y fortalece en su diseño la preocupación por el quehacer científico que fundamenta las acciones, razón por la cual se crea el Centro Estratégico del Análisis Delictivo. Además, reemplaza la unidad de interés para intervención de la política pública desde la comuna al barrio, "con el fin de focalizar y dirigir acciones de modo más certero y preciso, concentrando los recursos disponibles" (Lagos, 2012).

Otro elemento innovador, es el vínculo entre la adecuación de esta estrategia y la nueva institucionalidad con la cual ahora funciona en el Estado, lo que ancla a la política de seguridad a esta nueva estructura estatal: El Ministerio del Interior y Seguridad Pública, que debuta el año 2011, hito que se relaciona directamente con la creación de la Subsecretaría de Prevención del Delito. Esto es especialmente interesante en lo relacionado con el tema de la Rehabilitación y Reinserción Social, donde luego de su categorización como eje estratégico por parte de la Estrategia Nacional de Seguridad Pública, adquiere consolidación y adquiere herramientas para su implementación. En específico, la ley 20.502, del 21 de febrero de 2011, crea el Ministerio del Interior y Seguridad Pública y el Servicio Nacional para la Prevención y Rehabilitación del Consumo de Drogas y Alcohol, además de instalar algunas disposiciones transitorias al respecto.

Desde este convenio de trabajo intersectorial, se derivan conceptualizaciones de trabajo respecto de la Reinserción Social, donde se la entiende como "un proceso sistemático de acciones que se inician desde el ingreso de una persona a la cárcel, durante el período de cumplimiento de la condena y prosigue cuando la persona retorna a su vida en libertad" (Ministerio de Justicia de Chile, 2013). Esta etapa, según el Ministerio de Justicia (2013), incluye nivelación educacional, intervención psicosocial, capacitación e inserción laboral, además de actividades deportivas y culturales, buscando incidir efectivamente en los factores individuales y sociales que mediaron para que una persona se haya involucrado en actividades delictuales.

\section{Metodología}

La presente investigación tiene un carácter cualitativo y descriptivo, y está basada en las herramientas de análisis Grounded Theory (Strauss y Corbin, 2002) y el Frame Analysis (Goffman, 2006). La Grounded Theory es una metodología que permite un análisis descriptivo y relacional, a través de los procesos de codificación abierta, codificación axial y codificación selectiva, además de la tipificación de dinámicas implicadas y de los procesos relacionales.

Se propone la siguiente estructura analítica como procedimiento para el uso de la Grounded Theory.

La ventaja de la Grounded Theory, es que la construcción teórica surge a partir de los datos, es decir de una manera inductiva. Se ha considerado el uso de la presente técnica por su capacidad para realizar una investigación abierta y flexible, que permita considerar constantemente aspectos no previstos a priori y por basar su trabajo enfocado en la pregunta de investigación en ausencia de hipótesis que puedan dirigir o sesgar el proceso de construcción de categorías y relaciones, aspectos importantes dadas las características exploratorias de esta investigación.

El Policy Frame Analysis, en tanto, es una herramienta particularmente útil para poder estudiar las negociaciones sobre la realidad por parte de los actores sociales, técnicos y políticos en sus respectivos discursos, estableciendo los frames que orientan el diseño e implementación de las políticas públicas. De esta manera, a partir de este enfoque metodológico, se busca indagar cómo las distintas visiones sobre un problema político son negociadas, debatidas y combinadas, para la generación de una visión compartida al respecto.

La recolección y análisis de los datos se desarrolló en un contexto temporal transversal debido al carácter descriptivo que pretende abarcar la investigación. A razón de estas consideraciones, los datos se extraen de los discursos oficiales emitidos por los distintos actores involucrados, que representan la visión actual de la institución respecto de la 
Cuadro 1: Modelo de análisis basado en Grounded Theory

\begin{tabular}{lll}
\hline Nivel & Método & Unidad de información \\
\hline \hline \multirow{2}{*}{ Intra Discurso } & $\begin{array}{l}\text { Codificación Abierta } \\
\text { (Proceso descriptivo) } \\
\text { Codificación Axial } \\
\text { (Proceso relacional) }\end{array}$ & $\begin{array}{l}\text { Categorías, propiedades } \\
\text { Policy frame, relaciones (conflicto, contraposición, } \\
\text { dominación, etc.) }\end{array}$ \\
\multirow{2}{*}{ Inter Discurso } & $\begin{array}{l}\text { Codificación Axial } \\
\text { (Proceso relacional) }\end{array}$ & $\begin{array}{l}\text { Relaciones entre policy frame (Dominante, } \\
\text { complementario, alternativo, secundario, conflicto) }\end{array}$ \\
\hline \hline
\end{tabular}

Fuente: Elaboración propia

materia. En este sentido, la investigación se enmarca en atender a los Policy Frame actuales, tomando como referencia los últimos discursos emitidos por los distintos actores involucrados en este tema en el período 2012-2014.

\section{Universo y muestra}

En relación al objeto de estudio, se considera un amplio universo compuesto por aquellas instituciones involucradas en el trabajo con personas privadas de libertad en nuestro país, además de aquellos actores que tienen injerencia directa sobre el diseño de la política pública, los cuales tienen espacio en la construcción de significado en relación a este tipo de medidas.

Debido al carácter cualitativo de la presente investigación, se seleccionó una muestra definida en base a criterios de representación teórica (Hernández Sampieri et al., 2010). En razón de la factibilidad, la muestra se compone de los principales actores involucrados en la discusión sobre el problema de la Reinserción Social de privados de libertad en nuestro país. En términos de la operacionalización de los actores, consideramos lo que Bossaguet, Jacquot y Ravinet (2009) señalan, siendo considerado un actor relevante aquel capaz de llevar a cabo acciones estratégicas, planificadas y que tengan repercusiones directas en su definición, ya sea a través del proceso político en sí o en el resultado de dicha política.

De esta manera, los actores a considerar son: Fundación Paz Ciudadana, Gendarmería de Chile, Cámara Chilena de la Construcción, Subsecretaría de Prevención del Delito, Fundación Paternitas, Sodexo.

\section{Resultados}

\section{Caracterización de los Policy Frame por actor}

\section{Subsecretaría de Prevención del Delito}

El primer aspecto importante a señalar es el objetivo de la Subsecretaría de Prevención del Delito, el cual corresponde a intervenir sobre la victimización a través de la disminución del nivel de Riesgo de Reincidencia delictual, aspecto que crea un marco de trabajo conectado directamente con un modelo teórico de intervención integrado por 2 enfoques principales: el de Riesgo-Necesidad-Responsividad (Andrews, 2010); y el modelo Motivacional Transteórico (Prochaska y DiClemente), en el marco de la intervención basada en evidencia.

Su modelo de trabajo, contempla la intervención sobre Factores de Riesgo de Reincidencia Delictual intrapenitenciario y postpenitenciario, sumado al trabajo de Gestión de Casos individual postpenitenciario, enfocada en otorgar continuidad a la intervención basada en un plan de intervención individual que contempla lo psicológico, social y lo socio-ocupacional.

Lo anterior, deja en evidencia que la aproximación del condenado hacia la libertad es un proceso de aproximación progresivo, a través del uso de Beneficios Intra-Penitenciarios (BIP), con seguimiento de profesionales y coordinación con instancias post-penitenciarias.

Un aspecto al cual se le da relevancia, es que se considera la voluntariedad del usuario para trabajar en su Reinserción, y se trabaja su motivación como una constante a través de todo el proceso. Propone que la Reincidencia Delictual ocurre por la conjunción de Factores de Riesgo Estáticos y Dinámicos, para lo cual se debe enfocar la intervención en aquellos relacionados con el delito, así como habilitar al usuario en la construcción de un nuevo entorno so- 
cial y laboral a su egreso.

Considera una serie de herramientas de trabajo, que permitan alcanzar los fines antes señalados. Destaca un instrumento de evaluación del Riesgo de Reincidencia: el Ofender Assesment System (OASYS), el Plan de Intervención individual, Talleres estandarizados (Psicosociales y socio-ocupacionales) y el otorgamiento de libertad progresiva.

Todo lo anterior, fundamentado en el modelo de Intervención Basada en Evidencia, criterio respecto del cual, se escogen las herramientas e instrumentos de trabajo, se revisa su efectividad de manera constante, se corrigen y ajustan.

La mirada desde la Subsecretaría de Prevención del Delito, promueve un enfoque de intersectorialidad entre distintos participantes. En este sentido, propone la importancia de coordinar las instituciones públicas que se relacionan al tema y puedan aportar en la Reinserción de ex privados de libertad, además de recibir contribuciones desde el mundo privado. Busca la coherencia y consistencia entre las distintas intervenciones, la coordinación de los participantes para alcanzar cobertura, el acceso e intercambio fluido de la información sobre los usuarios entre estos.

\section{Gendarmería de Chile}

En primer lugar, se observa la presencia de un objetivo declarado siendo este el contribuir a la seguridad, desarrollando programas de Reinserción Social que tiendan a disminuir las probabilidades de Reincidencia Delictual. Para ello, propone el trabajo en distintos frentes de acción o subsistemas de intervención - Régimen Cerrado, Semi-abierto y Abierto-, criterio a través del cual organizan sus usuarios y distribuyen la oferta de intervención. Estos combinan criterios técnicos y de recursos para la atención de los usuarios condenados privados y ex privados de libertad.

Su población objetivo se encuentra organizada de manera distinta para los 3 Subsistemas, a través de criterios técnicos y basados en los recursos disponibles que organizan la modalidad de trabajo de estos 3 subsistemas.

En cuanto al Subsistema Cerrado, se mantienen prestaciones psicosociales generales a todos los usuarios, posibilidades de capacitación, empleo, actividad deportiva lúdica, educación y vigilancia, siendo la mayoría ofrecida ante la demanda del usuario excepto la última. El trabajo en este Subsistema, se considera el primer proceso de intervención que prepara al usuario que los demanda al acceso a permisos de salida.

El Subsistema Semi-abierto, incorpora también prestaciones de vigilancia, de perfilamiento (diagnóstico, plan de intervención, etc.) para la organización de los usuarios, prestaciones psicosociales a la demanda, talleres laborales para todos los usuarios, capacitación en oficios y nivelación de estudios. En este Subsistema, los usuarios cuentan con BIP, y es una alternativa para usuarios que cuentan con mejores condiciones para aproximarse al medio libre.

En el subsistema Abierto, encontramos como prestaciones el seguimiento (control de requisitos legales), gestión de redes (búsqueda de oferta según necesidades del usuario), intervención psicosocial (a través de un plan de intervención), nivelación de estudios, Inserción laboral y apoyo jurídico. Este subsistema corresponde a la alternativa que da continuidad a cualquiera de los dos subsistemas anteriores.

Este modelo de trabajo promueve la movilidad de los condenados que cumplan los requisitos a través de la obtención de BIP, para su aproximación paulatina a la libertad. Considera como centro de su trabajo la reducción de la Reincidencia Delictual de los egresados del subsistema cerrado, a través de la mejora de las oportunidades de integración social.

Lo anterior a través del fomento de conductas, habilidades y competencias que incrementen sus posibilidades de reincorporarse a la vida en sociedad. Para ello, declara buscar la inclusión de familias, instituciones, empresas y comunidad en general, en el proceso. No especifica enfoques de intervención específicos, a través de los cuales opera el modelo de trabajo.

Sin perjuicio de lo anterior, especifica algunas herramientas con las cuales operan de manera general los distintos subsistemas y los funcionarios encargados de su operación. Entre ellos, se encuentra el trabajo basado en planes de intervención individual, intervención psicosocial, seguimientos de casos (para control jurídico y apoyo psicosocial), apoyo jurídico y gestión de redes (a través del establecimiento de convenios).

En los tres subsistemas de trabajo, mantiene una visión de trabajo y colaboración intersectorial, el cual busca obtener prestaciones para los usuarios que la institución no ofrece o no puede ofrecer en la proporción que se necesitan para la cobertura de toda su población. 


\section{Fundación Paz Ciudadana}

Desde el punto de vista de Paz Ciudadana, en tanto Fundación que entre otras funciones elabora conocimiento y propuestas sobre Políticas Públicas en esta área, presenta como sugerencia y orientación (objetivo declarado para Reinserción Social), la disminución de los niveles de Reincidencia Delictual en nuestro país, los cuales a su juicio son altos y carecen del apropiado abordaje. Se manifiesta a favor y promueve la intersectorialidad, especificando los roles que cada uno de estos debiese cumplir.

Señalan, como población objetivo, aquella que se determine a través del diagnóstico de los distintos tipos de perfiles, determinando su Riesgo de Reincidencia (basado en instrumentos diseñados para ello), con foco en aquellos que presenten alto riesgo, $\mathrm{y}$ destinando un trabajo intensivo sobre el primer año de egreso, el cual muestra ser el de más alto Riesgo de Reincidencia.

Así, plantea que la ocurrencia de la Reincidencia Delictual es producto de la conjunción de Factores de Riesgo Estáticos y Dinámicos. Con ello, el modelo de trabajo sugerido es un sistema basado en aquellos que hayan mostrado ser más efectivos, lo que sugiere un enfoque de administración basado en evidencia. De esta manera, proponen el trabajo bajo el enfoque de intervención RNR (Andrews, 2010). Además, propone como herramientas de trabajo la evaluación a través de instrumentos que midan el Riesgo de Reincidencia, la Terapia Cognitivo Conductual, Terapia Multimodal, seguimiento, gestión de casos y redes.

El usuario deberá, a través de lo anterior, alcanzar una aproximación paulatina a la libertad, con apoyo post-penitenciario. En este sentido, será crucial desde la perspectiva de este actor crear programas transicionales para el egreso.

Este actor, realiza críticas y propone medidas indispensables a implementar para mejorar los sistemas de trabajo que observa en la actualidad. A modo de crítica, manifiesta la muy baja cobertura actual de los programas gubernamentales, la inexistencia de programas de Reinserción Social propiamente tal, ya que estos solo alcanzan estructuras intermedias que no alcanzan a conformar programas (Falta de integridad), inexistencia de oferta especializada para delitos sexuales, violentos o contra la familia (VIF).

En relación a la falta de integridad de los programas, señala criterios que están presentes, ausentes y parcialmente incorporados en la oferta actual: calidad profesional, relación con el usuario, documentación del programa, roles definidos, accountability, sostenibilidad, entre otros.

Por otra parte, propone la creación de un Servicio Nacional de Reinserción en el Medio Libre, que oriente y organice el trabajo en reinserción, el rediseño de la oferta post-penitenciaria, la creación de oferta especializada para infractores en delitos sexuales y VIF, establecer e iniciar la implementación de un plan de expansión para lograr la cobertura.

\section{Fundación Paternitas}

La Fundación Paternitas, es un organismo que desarrolla programas de intervención para ex privados de libertad, vinculado a la iglesia católica, cuya presidencia se encuentra a cargo del sacerdote diocesano Nicolás Vial Saavedra, quienes además su fundador. Propone, como objetivo de sus intervenciones, el disminuir la Reincidencia Delictual, su impacto en la sociedad y en las familias de los ex privados de libertad, a través de la Reinserción Social.

Para ello, considera crucial el trabajo intersectorial como forma de trabajo conjunto entre el sector público y el privado, para facilitar la articulación de los programas de reinserción de la fundación y el mercado laboral.

Plantea sus focos de intervención, en las personas ex privadas de libertad, primerizos o próximos a cumplir condena y sus familias. Para ello, abordan el trabajo con varones adultos, de la red de salud occidente (Santiago) y/o que hayan sido derivados de tribunales de alcohol y drogas o de forma particular. Además, trabaja con hijos de ex privados de libertad, niños que hayan sido vulnerados, y familias de los anteriores.

La Reincidencia Delictiva, es entonces producto de factores relacionados con la cultura del individuo, donde la privación de una socialización adecuada (educación, patrones transgeneracionales) y el problema de las drogodependencias son factores gravitantes.

Sugieren apoyar a los ex privados de libertad para sanar sus heridas espirituales, emocionales, psicológicas y neuropsiquiátricas que impidan su retorno a la sociedad. Para ello, la principal actividad que permite al individuo re-vincularse con la sociedad en patrones sociales distintos, es el desarrollo de una historia laboral que permita la rehabilitación y reinserción social de la persona y su grupo familiar.

Lo anterior, se efectúa a través de un modelo de trabajo basado en 3 elementos: El trabajo de intervención Psicosocial y socio-ocupacional con el 
ex privado de libertad, trabajo con sus familias y el trabajo en drogodependencias con quienes lo requieren.

En términos generales, su propuesta se fundamenta en un enfoque de competencias prosociales. En relación a estos, se utilizan diversas herramientas que permiten el trabajo en sus objetivos declarados, entre las que se encuentra: la intervención psicosocial, la habilitación socio-ocupacional, la capacitación en oficio, talleres individuales y grupales (productivos, de inclusión social, alfabetización, recreación y desarrollo personal), el seguimiento, orientación jurídica (individuo y familia), la comunidad terapéutica (drogodependencias) y el tratamiento neuropsiquiátrico. No sugieren específicamente un enfoque para el trabajo de intervención, así como tampoco establece específicamente una modalidad para que el individuo alcance la libertad.

A modo de crítica, manifiesta la prioridad que Gendarmería tiene en cuanto al resguardo de la seguridad en desmedro de la Reinserción Social y la importancia de la Reincidencia Delictual para la sociedad. Adicionalmente, señala el pobre éxito de los programas existentes donde calcula un tasa de un 8.9\% aproximado de inserción. En relación a esto, propone la apertura de Gendarmería y SENAME al trabajo con privados y ONG's que puedan ofrecer prestaciones o modelos de trabajo más efectivos que los propios.

\section{Cámara Chilena de la Construcción}

La Cámara Chilena de la Construcción (CChC), plantea como objetivo declarado producir la inserción socio-laboral de las personas más vulnerables de la sociedad en la fuerza productiva del país. Esto incluye el trabajo con ex privados de libertad, por lo que ha originado el Programa Cimientos, a través del cual buscan el objetivo antes señalado para este tipo de población. Su población objetivo es masculina, mayor de 18 años, y que cuente con aspectos que permitan proyectar éxito viable luego del trabajo realizado.

Desde la CChC, se conserva una mirada prudente, visualizando el trabajo a través de su programa como una intervención de carácter complementaria a las ofrecidas por programas que se dedican a esto. En relación a ello, promueve un trabajo que conecte el sector público y privado a través de las políticas públicas, basado en la importancia de fomentar la participación de la ciudadanía en la generación de nuevas formas de subsistencia y bienestar social. Destaca además, la eficiencia demostrada por el sector privado para favorecer el desarrollo.
En cuanto a su modelo de trabajo, destaca la inserción laboral y social con una oferta de 4 acciones concretas para su implementación: la formación para el trabajo, la capacitación en un oficio del sector construcción, la colocación laboral, el monitoreo y seguimiento de los casos.

Para este actor, el alcance de la libertad, debe ser a partir de la aproximación paulatina al medio libre, con uso de los distintos BIP existentes para la incorporación a programas de habilitación e inserción en el campo laboral. Para ello, utiliza instrumentos de trabajo relacionados con la capacitación laboral y en competencias sociales, la colocación laboral, el apoyo psicológico, lo seguimientos y orientaciones post-egreso. Parte de esta forma de trabajo es la importante vinculación del programa con instituciones laborales que puedan facilitar la práctica e inserción de sus usuarios.

Este actor, efectúa una crítica a Gendarmería, basada en que su intervención se focaliza en la seguridad, desplazando su rol de rehabilitación e inserción social a un segundo plano, lo que dificulta el acceso a los potenciales beneficiarios y entorpece la ejecución de proyectos.

\section{Sodexo}

Sodexo, a través del manejo de cárceles concesionadas, ha destinado el trabajo de su departamento Justice Services, al trabajo en la administración de cárceles y con ello, bajo la normativa legal chilena, adquiere el compromiso de trabajar el ítem de Reinserción Social. Para ello, trabaja la adquisición de competencias prosociales, de competencias para el desempeño de un oficio, el respeto de las normas de vida colectiva, la valoración personal, el respeto hacia sí mismo y los demás, otorgando así, las condiciones individuales y de contexto para su Reinserción efectiva.

Declaran explícitamente que su población objetivo es aquella condenada y recluida en sus establecimientos penales, sin señalar otras consideraciones específicas. Considera el alcance de la libertad a través de un proceso de aproximación paulatina al medio libre, mediante el uso de BIP para la habilitación e inserción en el campo laboral.

Propone como modelo de trabajo el abordaje a través de dos estrategias principales: las competencias prosociales y la educación. En el primer caso, la describe como un programa de carácter multifacético, con enfoque cognitivo conductual, que instaura competencias y habilidades sociales en los internos, lo que los ayuda a establecer un proyecto de vida prosocial y respetuoso de las normas sociales. En el 
segundo, programas educacionales, de capacitación y formativos de habilidades para el trabajo.

Sodexo, se muestra a favor del trabajo intersectorial, promoviendo la conformación de alianzas estratégicas con instituciones que agreguen valor a la actividad, sobre todo, en la generación de puestos de trabajo para el egreso de los privados de libertad.

Este actor, no se hace presente con críticas ni propuestas explícitas en cuanto a la organización del trabajo en materia de políticas públicas de Reinserción Social.

\section{Análisis intra Frame.}

A continuación se presentan los análisis efectuados luego de la recolección de datos provenientes de los distintos actores examinados. En el cuadro 2 y en el cuadro 3 , se presentan los criterios para el análisis de datos.

\section{Usuario objetivo}

En cuanto al usuario objetivo del trabajo en Reinserción Social, los distintos actores confluyen en considerar el trabajo con personas condenadas mayores de edad, con lo que se excluyen los imputados y menores de edad (jurisdicción de SENA$\mathrm{ME}$ ), quienes no son sujeto de este tipo de intervenciones. Para esto, es importante el trabajo tanto con privados de libertad que se acerquen al egreso en términos de tiempo de cumplimiento de condena, además de aquellos que se encuentran con BIP o egresados desde hace poco.

Esto configura el Frame Hegemónico respecto del usuario, el cual tiene basamentos técnicos (organización penal e inserción), legales (administración de BIP y requisitos) y administrativos (uso de recursos focalizado) fundamentalmente.

Se observa como Frame Complementario compartido por Paz Ciudadana, Paternitas y la Subsecretaría de Prevención del Delito, la selección de usuarios fundamentado en criterios técnicos que permitan organizar la población, como por ejemplo: Riesgo de Reincidencia, Compromiso Delictual, Conducta, Salud mental, entre otros, lo que se inscribe en una forma de trabajo que considera perfiles específicos de tratamiento, en relación a la intervención basada en evidencias, así como en el modelo de RNR.

Por otro lado, se observa como complemento la visión de $\mathrm{CChC}$, quien incorpora como criterio la viabilidad de los sujetos a cumplir con la interven- ción y apoyos, aspecto relacionado con la cobertura que este actor puede entregar y la relación entre el uso de los resultados de la intervención y la mantención de los recursos para funcionar (privados que aportan en función de los resultados), lo que se realiza a través de un diagnóstico técnico que permita la proyección del usuario a través de las actividades de habilitación y capacitación.

Paternitas y Gendarmería añaden diferencias que no pueden ser entendidas como Frame, puesto que son limitaciones que cada uno cuenta como institución, más que una visión respecto de cómo se debe realizar la actividad, pero que sin embargo son relevantes de comentar.

Paternitas, incorpora un criterio de selección geográfica que filtra los usuarios de un sector específico (por tema de recursos y redes). Por su parte, Gendarmería se rige a partir de criterios basados en limitaciones de recursos y exigencias legales, las cuales solo aplican a este caso específico (obligaciones en tanto institución encargada del estado de garantizar Reinserción Social).

\section{Foco para la Reinserción Social.}

Con respecto al foco para la reinserción social, los distintos actores confluyen en la importancia de considerar como foco de trabajo el factor Reincidencia Delictual.

Tal como se observa en la literatura al respecto, una de las principales preocupaciones de los distintos actores es el cómo evitar la Reincidencia en el delito, en tanto que esta incrementa la Inseguridad Ciudadana, además de contribuir a fortalecer los factores de riesgo estáticos - invariables - y de profundizar la cultura criminal del involucrado, elementos muy vinculados a las perspectivas integrales de abordaje del delito revisadas previamente (Modelo RNR).

A pesar de este foco común, existen diferencias importantes de considerar por parte de cada actor, que pueden ser considerados Frames Complementarios, donde la victimización e impacto social emergen como importantes puntos de convergencia. En este punto, es interesante observar como convergen actores con alta vinculación al Estado, lo que puede ser un efecto de la arraigada cultura del control y la punición en este sector.

Paternitas, agrega la importancia de no solo considerar las víctimas, sino que también centrarse en la vinculación de la Reincidencia como un factor que perjudica a quienes la efectúan (ex-privados de libertad) y a sus familias, siendo este el sello 
Cuadro 2: Criterios para el análisis de datos

\begin{tabular}{ll} 
Criterios & Descripción \\
\hline \hline Usuario objetivo & $\begin{array}{l}\text { Personas sujeto de las intervenciones a realizar, y por lo tanto, } \\
\text { de la política pública. }\end{array}$ \\
Conceptualización Problema-Solución & $\begin{array}{l}\text { Visión del problema sobre la Reinserción Social } \\
\text { y de sus posibles soluciones. }\end{array}$ \\
Modelos de intervención & $\begin{array}{l}\text { Modelos teóricos que sostienen las intervenciones propuestas } \\
\text { por los distintos actores. }\end{array}$ \\
Herramientas para la gestión & $\begin{array}{l}\text { Instrumentos que los distintos actores relevan al momento } \\
\text { de operar para el trabajo en Reinserción Social. }\end{array}$ \\
Articulación entre Actores & $\begin{array}{l}\text { Visión acerca de la interrelación que deba existir entre los } \\
\text { distintos actores involucrados en Reinserción Social. }\end{array}$ \\
Acceso a la libertad & $\begin{array}{l}\text { Establece la manera en que los distintos actores conciben } \\
\text { la aproximación del privado de libertad al medio libre. }\end{array}$ \\
Foco para la Reinserción Social & $\begin{array}{l}\text { Referido a los aspectos que los distintos actores consideraron } \\
\text { centrales como parte de la Reinserción Social. }\end{array}$ \\
\hline \hline
\end{tabular}

Fuente: Elaboración propia

Cuadro 3: Criterios para el análisis de datos

\begin{tabular}{ll}
\hline Tipología & Descripción \\
\hline \hline Frame Hegemónico & Frame predominante, compartido por la mayoría de los actores.
\end{tabular}

Frame complementario

Frame presente en la visión de uno o más actores, que se aprecia como un complemento al Frame Hegemónico.

Antagonismo de frames Frame presente en la visión de uno o más actores, que representa oposición o incompatibilidad con alguno de los frames presentes.

\footnotetext{
Fuente: Elaboración propia, basado en criterios utilizados por Martin (2012) para su investigación.
}

distintivo y exclusivo de esta fundación.

$\mathrm{Al}$ respecto, se observa una relación entre este tipo de énfasis producto de la naturaleza de la institución - Católica, dirigida por un Sacerdotedonde se combinan fines doctrinarios con elementos técnicos, que justifican la inclusión en el foco de intervención a las "heridas" del delincuente, debido a su estilo de vida, así como las de sus familiares.

\section{Conceptualización problema-solución.}

En cuanto a la conceptualización del problema que los distintos actores efectúan, se observa convergencia entre todos los actores en la falta de trabajo como un importante factor desencadenante y mantenedor de la Reincidencia, en tanto que la ha- bilitación/capacitación aparece como importantes elementos para su reintegración a la sociedad.

Desde la Subsecretaría de Prevención del Delito y Paz Ciudadana, surge el enfoque de RiesgoNecesidad-Responsividad (Andrews, 2010), el cual se perfila como principal modelo, que desde una perspectiva integrativa e interdisciplinaria, permita explicar la delincuencia e intervenir infractores de ley.

Este modelo es planteado por los 3 actores que se encuentran en directa relación con las perspectivas más sofisticadas y de vanguardia a nivel mundial en cuanto a Rehabilitación y Reinserción, cuya naturaleza y origen se suscribe a la necesidad de enfoques científicos basados en evidencia respecto de su efectividad. 
Gendarmería por su parte, manifiesta su adscripción implícita al considerar el Riesgo de Reincidencia y enfoques de intervención amparados en esta concepción, a pesar de no ser declarativos al respecto.

La Subsecretaría de Prevención del Delito, adscribe además al enfoque Transteórico Motivacional (Proschaska y DiClemente), como fuente transversal de la intervención sobre la motivación de los individuos, de modo de establecer distintos momentos de intervención y de catalizar los distintos estados motivacionales. Este enfoque es propuesto en complemento al modelo RNR, y permitiría explicar la adherencia de los sujetos al tratamiento, así como facilitar los procesos de intervención.

Por otro lado, los actores Paternitas y SODEXO, fundamentan su trabajo principalmente en el enfoque de las Competencias Prosociales, lo que ocasiona antagonismo de frames entre las visiones sobre el problema-solución desarrolladas por este grupo y el grupo antes señalado.

Es por esta razón que no existe un Frame Hegemónico en la conceptualización sobre el problema-solución.

\section{Modelo de intervención}

En relación al uso de herramientas de intervención, existe un frame hegemónico que aglutina las formas de intervención para los privados de libertad de cara a su Reinserción en la sociedad: Intervención multimodal, inserción en el entorno social, apoyo y seguimiento.

Es así como confluyen todos los actores en la necesidad de incluir planes de intervención individual, el trabajo a través de terapia cognitivo conductual, la entrega de orientación social y jurídica en las distintas fases del proceso, el trabajo a través de talleres grupales psicológicos y sociales, además de talleres grupales recreativos y socio-ocupacionales, con oferta de nivelación y educación formal, los que se enmarcan en la forma de intervención multimodal o multisistémica.

Además, los actores convergen en la necesidad de ofrecer colocación laboral a quienes cumplan con el proceso, y de ofrecer seguimiento y apoyo. Siendo a través de estos elementos que se construye el enlace con el medio libre, circunscritos en la forma de inserción, apoyo y seguimiento.

Todo lo anterior, sin perjuicio, de que no todos los actores utilizan exactamente las mismas herramientas, variando en función de la perspectiva teórica, el tipo de usuario y la extensión temporal en que buscan abordar.

Esta visión sobre el tratamiento del delincuente, plantea la necesidad de un encadenamiento en el proceso de intervención donde la Reinserción Social aparece como la continuidad de la Rehabilitación efectuada en el medio libre.

Lo anterior, sitúa la Reinserción social en base a un modelo de trabajo integrativo e interdisciplinario, centrando sus acciones en el delincuente y su entorno, considerando la intervención intrapenitenciaria, en el tránsito hacia la libertad, así como en libertad (seguimientos).

Adicionalmente, encontramos para la Fundación Paternitas, como una herramienta distintiva, la Intervención a los ex-privados de libertad desde la perspectiva Neuropsiquiátrica, incorporando técnicas de este enfoque, lo que se sitúa de manera complementaria, siendo desde la perspectiva de este autor un elemento crucial en su abordaje de la intervención.

Esta perspectiva, basada en el modelo biomédico, escapa a la tendencia general de abordaje psicosocial, lo que establece otro punto de divergencia de este actor en relación a los demás.

En general, los distintos actores confluyen en la idea de mantener una evaluación diagnóstica que perfile y organice los distintos tipos de necesidad de los usuarios para la intervención.

Sin embargo, Paz Ciudadana y la Subsecretaría de Prevención del Delito, especifican el uso de escalas estandarizadas para la evaluación, basadas en RNR, con muy baja discrecionalidad profesional, mientras que los demás actores dejan este elemento al criterio del profesional, quien debe utilizar como base el modelo específico que la institución promueve, con bastante discrecionalidad profesional.

Lo anterior, ocasiona antagonismo de frames en este punto específico, donde si bien existe consenso en cuanto a la utilidad diagnóstica y de perfiles para el trabajo de intervención entre todos los actores, existe una discrepancia específica entre 2 actores y los restantes, respecto del trabajo bajo escalas estandarizadas $\mathrm{v} / \mathrm{s}$ la discrecionalidad profesional, ocasionando incompatibilidad técnica entre estas visiones.

\section{Herramientas para la gestión}

Los actores, confluyen en este aspecto, en relación a la Gestión con Redes tanto públicas como privadas para la obtención de prestaciones de distintos orden, que por sí mismo no son capaces de 
proveer o que exceden sus ámbitos de desempeño, restricciones legales $\mathrm{u}$ otros, siendo este el frame hegemónico para este tópico.

Parte de esta misma visión, que permite la incorporación de agentes privados en la prestación de servicios asociados a la delincuencia, se encuentra la incorporación de OTEC y OTIC como agentes relevantes de oferta en capacitación y colocación laboral, la llegada de cárceles concesionadas (SODEXO), el involucramiento de Fundaciones tanto en estudios como servicios, todo lo anterior en el trabajo intrapenitenciario, de transición hacia la libertad, de apoyo y de seguimiento.

Asimismo, es compartida la idea de la utilización de BIP para la aproximación a la libertad, y como instrumento legal para la articulación con otras redes de trabajo, con excepción de Paz Ciudadana que no se pronuncia en este aspecto específico (Frame Complementario).

Estos aspectos, se encuentran conectados al contexto de desarrollo de la legislación, donde algunos instrumentos legales permiten pensar en la incorporación de elementos del paradigma restaurativo, como por ejemplo la ley de Libertad Condicional, ley de Rebaja de Condena y por supuesto, programas ministeriales asociados a Rehabilitación y Reinserción Social, entre otros.

De manera, las fundaciones Paz Ciudadana y Paternitas, así como la Subsecretaría de Prevención del delito, concuerdan en relación al enfoque basado en evidencias, es decir, administración de programas que hayan tenido buenos resultados de manera consistente a través de distintas investigaciones, enfoque de gestión que implica también una postura en cuanto a la administración de recursos necesarios, la que se gerencia desde la mirada técnica.

Esto se corresponde con la visión internacional respecto de la criminalidad, donde diversos autores, entre los que destacan Andrews y Bonta (2010), han mostrado la poca efectividad de los sistemas de tratamiento para lograr efectos en la intervención, influyendo directamente en la mirada del ministerio del Interior, donde los enfoques son estrictamente basados en evidencia científica, además de considerar un riguroso accountability para dichas actividades.

Otro Frame Complementario, guarda relación con la gestión de educación formal con instituciones de educación públicas acreditadas para la función, visión sostenida por Gendarmería, la Subsecretaría de Prevención del Delito y Paternitas, quienes resguardan aún esta función como prioridad del sistema público educacional Chileno con sus distintas modalidades.

\section{Articulación entre actores}

En relación a este ítem, es importante señalar la confluencia de todos los actores para favorecer la interconexión entre los distintos actores involucrados en el trabajo sobre Reinserción Social (motivo por el cual existen múltiples convenios de colaboración), aspecto que mantiene la lógica de funcionamiento antes señalada, respecto de la incorporación de actores privados en distintos procesos, siendo función exclusiva de Gendarmería la administración y gestión penitenciaria, además de la implementación de la penalización.

Sin embargo, difieren en aspectos específicos relacionados con la finalidad de este trabajo y sus características. Aparecen 4 frames complementarios, compartidos por diversos actores. Sus ejes son: coherencia y consistencia en el trabajo realizado por distintas las instituciones, la cooperación público privada para encontrar articulación con el mercado laboral, alianzas entre instituciones para agregar valor y políticas públicas para incluir al privado en la oferta de prestaciones en Reinserción Social.

De la misma manera, se observan frames complementarios que no son compartidos con otros actores: fluidez en el acceso e intercambio de la información, coordinación entre aquellos actores que entregan prestaciones distintas para la intervención, cooperación público-privada para la articulación de las prestaciones y trabajo intersectorial para la cobertura de prestaciones que no entrega.

Por otra parte, se observa antagonismo de frames sostenido por 3 actores, quienes consideran que existe una especificación concreta de los roles públicos y privados: El rol público será entregar directrices de funcionamiento y delimitar los marcos legales de trabajo, mientras que el privado debe aportar con prestaciones y complementes que el público no es capaz de brindar, especialmente en la articulación del usuario a con el medio libre.

\section{Acceso a la libertad}

En relación a la posición declarada respecto del método de aproximación a la libertad, se observa correspondencia de las visiones entre actores, en cuanto a favorecer la aproximación paulatina del condenado hacia la libertad, a través de los Beneficios Intra-Penitenciarios y en observación de los resultados de la intervención en curso, lo cual corresponde al Frame Hegemónico, lo que nuevamente se encuentra en conexión con la aproximación a un paradigma restaurativo y la apertura a enfoques que incorporan progresos en el tratamiento de la delincuencia. 
Se observa como frame complementario compartido por la Subsecretaría de Prevención del Delito y Paz Ciudadana, la importancia de programas transicionales que permitan el ajuste del condenado al medio libre. Estos deben incorporar apoyo y seguimiento post egreso, y coordinación con Redes de Apoyo post-penitenciarias y/o sociales, aspecto en total coherencia con la visión internacional en cuanto al tratamiento de la delincuencia.

Por otro lado, se observa como frame complementario compartido por SODEXO y CChC, la visión acerca de la necesidad de la aproximación paulatina para lograr inserción y habilitación laboral, en tanto que una de los aspectos principales que promueven estos actores para el tratamiento de la delincuencia es el trabajo. El foco es la responsabilización individual de los sujetos respecto de su proceso de cambio y Reinserción, encontrando fundamentos en los modelos de ruptura de vínculos sociales y el enfoque integrativo para el tratamiento del delincuente, basado en factores de riesgo de reincidencia.

Por último, Gendarmería tiene un frame complementario, que refiere al favorecimiento del egreso de los condenados, a través de los BIP basado en requerimientos y exigencias de carácter legal, aspecto circunscrito a esta institución basada en sus características fundacionales, su dependencia jerárquica (Ministerio de Justicia), así como en su enfoque de trabajo basado en el control, y más actualmente, en el potenciar conductas y habilidades prosociales del individuo, que incrementen las posibilidades de Reinserción.

\section{Conclusiones}

El primer punto importante de señalar, es que en esta investigación fue posible identificar distintos Policy Frame a la base de las propuestas sobre el tema de la Reinserción Social así como en la observación de sus actividades y propuestas programáticas. Los Policy Frame se fueron identificando a partir de información que no solo provino de aspectos declarados textualmente, sino que también de una combinación de análisis extraídos de las propuestas de trabajo, los métodos y los resultados de intervención de cada uno de los actores.

A partir del análisis axial inter-frame, fue posible verificar distinto grado de conexión e interdependencia, entre los Policy Frame de los distintos actores, y en este sentido, distintas relaciones. Se aprecian ejes comunes a todos los actores (Frame Hegemónico), visiones que complementan los ejes comunes sea desde la visión particular de un actor y/o la visión compartida por distintos actores (Frame Complementario), además de contraposiciones entre las distintas visiones (Frames antagónicos).

En cuanto al Frame Hegemónico, aparecen aspectos indispensables en la construcción del relato sobre la Reinserción Social, por parte de los distintos actores involucrados en la temática, lo que en parte, permite el diálogo y la posibilidad de intercambio de ideas entre estos, en incluso la oposición (antagonismo) en algunos puntos particulares.

Este núcleo se encuentra hilvanado y articulado por el modelo Riesgo Necesidad Responsividad (RNR) y su conceptualización de la delincuencia, donde los Factores de Riesgo, a través de elementos Estáticos (sin posibilidad de cambio favorable) y Dinámicos (con posibilidad de cambio favorable), permiten pensar en la oportunidad de cambio de la población privada de libertad y las condiciones mínimas para ello. Las necesidades criminógenas permiten integrar la resiliencia y las características individuales de los sujetos, para que se vinculen a Factores de Riesgo, facilitando o dificultando el involucramiento en actividad delictual. Por su parte, la Responsividad permite comprender la importancia de una motivación cíclica, la cual puede ser influenciada por modelos de intervención efectivos, y la que posibilita, a través de intervenciones pertinentes y atingentes, el cambio de la población penal.

Adicionalmente, este núcleo de comprensión sobre el problema de la delincuencia permite especular el rol estatal y social concebido respecto de la delincuencia y el tratamiento de la población penal, donde aparece un carácter restaurativo del Estado, su alta responsabilidad para con la temática, con un involucramiento parcial del sector privado.

$\mathrm{Al}$ respecto, es importante destacar la presencia de Frame Hegemónico para todas las temáticas, que si bien no son suficientes para entregar una respuesta profunda y acabada ante cada ítem, permiten consensuar una visión común entre todos los actores que enmarca un escenario trabajo común.

Existe una gran variedad de Frame Complementarios promovidos por algún actor, los cuales permiten profundizar los Frames Hegemónicos. A través de estos, cada actor detalla los matices que los diferencian, a la vez que permiten identificar con claridad sus modelos de funcionamiento.

De la misma manera, existe variedad de Frames Complementarios compartidos por distintos actores, los cuales también agregan matices específicos propios de cada actor, además de permitir observar los elementos que congregan a distintos actores en visiones comunes respecto de los distintos ítems. 
Por otra parte, existen algunos Frames antagónicos, los que reflejan una visión particular o compartida sobre un aspecto en específico que se contrapone a otra visión presente.

Para el ítem Visión sobre el problema-solución, la contraposición de modelos explicativos y de intervención (RNR v/s Competencias Prosociales), puede ser origen de tensión entre los actores involucrados y, con ello, permitir dificultades en una posible articulación futura. Si se observa en detalle, esta diferencia genera un vacío en cuanto a la predominancia de un modelo explicativo, en tanto que el Frame Hegemónico (Enfoque socio-ocupacional), no es suficiente para dar cobertura al proceso de Reinserción Social por sí solo (hace falta un modelo de intervención integral), y por lo tanto, facilita la generación de un área de conflicto (posicionar un modelo de intervención).

En el caso particular del ítem Modelo de Intervención, la contraposición existente centra el debate entre la mantención de un instrumento de Evaluación Estandarizado sobre Riesgo de Reincidencia que permita el trabajo en Reinserción Social, frente a una postura que promueve el perfilamiento de usuarios en base a los criterios del modelo de intervención que la institución prefiera.

Esta contraposición, ocasiona un vacío con respecto a la manera común de evaluar a los usuarios, lo que provee espacio para el conflicto y permite una brecha para la lucha por el posicionamiento de alguno de los modelos.

Se observa otra oposición de frames en el ítem Articulación de Actores, en donde no existe una visión predominante respecto de la articulación público-privada. Al respecto, la tensión aparece entre una posición que promueve la existencia de roles bien definidos para el sector público y el privado, mientras que la otra posición promueve que el privado pueda participar con libertad en todos los asuntos concernientes a Reinserción Social. Nuevamente, la brecha permite el conflicto para determinar cuál de estas visiones (u otra) deberá predominar al momento de ser necesario articular el trabajo o aunar criterios.

Desde las políticas públicas y las restricciones legales, existen espacios que permiten versatilidad en los frames sobre Reinserción Social, así como en los objetivos y acciones que los distintos actores puedan ofrecer a la población en cuestión, sobretodo en el sector privado (teniendo en consideración los estrictos lineamientos de la Subsecretaría de Prevención del Delito y las restricciones legales de Gendarmería de Chile).

A partir de la información recopilada, ha sido po- sible observar el grado de asociación entre los frames de los actores, teniendo en consideración la cantidad de aspectos que estos mantienen en común, lo que permite también observar la alineación que existe entre estos.

En este punto es importante señalar la relación observada entre Gendarmería de Chile, la Subsecretaría de Prevención del Delito y Paz Ciudadana, quienes comparten 3 ejes idénticos de trabajo, adicionales al Frame Hegemónico.

Por su parte, la Subsecretaría de Prevención del Delito, Paz Ciudadana y Paternitas, confluyen en un eje común de trabajo adicional, al igual que Gendarmería, Subsecretaría de Prevención del Delito y Paternitas.

Estos aspectos ponen en proximidad y alineación a estos actores, lo que puede ser un factor favorecedor para el trabajo articulado, además de otorgar fuerza a ideas y alianzas que surjan desde las perspectivas compartidas

Respecto de los actores individuales, es importante señalar la alta correlación que existe entre los frames promovidos por la Subsecretaría de Prevención del Delito y Paz Ciudadana, en donde se encuentra el más alto número de congruencias. Con ello la posibilidad de producir articulación, de recibir apoyo en ideas que surjan desde los frames comunes y de generar alianzas, se incrementa.

Por otro lado, se observa un alto grado confluencia con otros actores por parte de la Subsecretaría de Prevención del Delito, de Paternitas y de Paz Ciudadana.

En virtud de todo lo antes expuesto, se dejan entrever algunas cuestiones importantes de considerar luego de la observación y análisis de los policy frames de distintos actores relacionados con Reinserción Social.

En primer lugar, al observar la diversidad de Frame Complementario y el surgimiento de Antagonismos, cabe preguntarse acerca de la actual coordinación y alineamiento entre los distintos actores, así como los efectos que su congruencia o divergencia puedan traer al trabajo en la temática. En este sentido, cabe también preguntarse qué tan beneficioso pueda ser el establecimiento de precisos lineamientos y modelos top-down, con el actual modo de funcionamiento, en que parecen existir tenues líneas directivas desde los Ministerios (Justicia e Interior) y bastante libertad privada para la acción.

Otro tema de discusión, es la observación de distintas brechas de oportunidad para la aparición de conflicto, sea este para mantener los propios frames, 
o para generalizarlo como uno Hegemónico. En este sentido, será importante discutir acerca del rol que el Estado tenga en cuanto a la definición de las directrices de trabajo y las Políticas Públicas que permitan su implementación. Una discusión pendiente tiene lugar en cuanto a la necesidad o ventaja de homogenizar y/o circunscribir el trabajo realizado en esta temática frente a la libertad actual existente en cuanto al direccionamiento de los programas de Reinserción Social.

A la ya señalada tensión entre la libertad de acción y el direccionamiento top-down, se suma la discusión sobre la implementación de un enfoque basado en evidencias, así como la gestión basada en resultados, es decir, la preferencia por modelos de trabajo que han probado efectividad. Con ello, surgen cuestiones como ¿Cuáles son las mejores maneras de intervenir y gestionar? ¿Son estos modelos capaces de proveer buenos resultados en nuestro contexto? ¿Mediante qué sistema se debiesen decidir los lineamientos de trabajo en Reinserción Social?

\section{Referencias}

Andrews, D. A. (2010). The psychology of criminal conduct. Lexis Nexis, New Providence.

Banco Interamericano De Desarrollo (2006). La política de las políticas públicas. En Progreso económico y social en América Latina. Editorial Planeta, Washington.

Boussaguet, L., Jacquot, S., Ravinet, P., Cuervo Restrepo ed, J. I., Jolly ed, J.-F., y Soto Uribe ed, D., editores (2009). Diccionario de políticas públicas. Universidad Externado de Colombia, Bogotá.

Bustelo, M. y Lombardo, E. (2005). "Mainstreaming" de género y análisis de los diferentes "marcos interpretativos" de las políticas de igualdad en Europa: el proyecto MAGEEQ. Aequalitas: Revista jurídica de igualdad de oportunidades entre mujeres y hombres, (17):15-26.

Bustelo, M. y Lombardo, E. (2006). Los 'marcos interpretativos' de las políticas de igualdad en Europa: conciliación, violencia y desigualdad de género en la política. Revista Española de Ciencia Política, (14):117-140.

Cuvardic, D. (2001). Los marcos interpretativos en la ciencia social. Reflexiones, 80(1):3.

Escobar, A. (2008). Políticas públicas y penitenciarias: percepciones y efectos en un grupo de jóvenes penalizados: experiencia piloto en el centro de reinserción social de Angol. Tesis doctoral, Universidad de Chile.

Fischer, F. (2003). Reframing public policy : discursive politics and deliberative practices. Oxford University Press, Oxford.

Goffman, E. (2006). Frame analyisis: los marcos de la experiencia. CIS, Madrid.

Hernández Sampieri, R., Fernández Collado, C., y Baptista Lucio, P. (2010). Metodología de la investigación. Editorial Mc Graw Hill, México D. F.

Hidalgo, P. (2001). Las Políticas Públicas: Los Actores Principales y su Racionalidad. Observatorio de Políticas Públicas.

Jones, C. (1970). An introduction to the study of public policy. University of Pittsburgh, Duxbury.

Lagos, R. (2012). Políticas de seguridad, la experiencia chilena (2003-2011). Revista Análisis Público, 1:211-233.

Lahera, E. (2002). Introducción a las políticas públicas. Fondo de Cultura Económica, Santiago.

Martin, M. P. (2012). El cambio en el sistema de protección social en Chile: un análisis de policy frames. 3er Encuentro de la Sociedad Chilena de Políticas Públicas.

Meltsner, A. (1992). La factibilidad política y el análisis de políticas. Miguel Angel Porrua, México D. F.

Ministerio de Justicia de Chile (2013). Quiénes somos.

Morales Peillard, A. M. (2012). La política criminal contemporánea: Influencia en Chile del discurso de la ley y el orden. Política criminal, 7(13):94146.

Pantoja, R., Weinborn, C., Guzmán, G., y Acevedo, A. (2009). Programa de intervención psicosocial especializado en factores de riesgo dinámicos: Manual general del módulo. Salviat impresores, Santiago.

Roth, A.-N. (2002). Politicas públicas: formulación, implementación y evaluación. Eds. Aurora, Bogotá.

Santibañez, A., Barra, M., y Ortiz, P. (2013). La racionalidad de los actores en políticas públicas: Un esquema teórico para entender el funcionamiento de las democracias.

Schön, D. A. y Rein, M. (1995). Frame reflection: Toward the resolution of intractable policy controversies. Basic Books. 
Strauss, A. L. y Corbin, J. (2002). Bases de la investigación cualitativa: técnicas y procedimientos para desarrollar la teoría fundamentada. Universidad de Antioquia, Medellín.

Subirats, J., editor (2008). Análisis y gestión de políticas públicas. Ariel, Barcelona.

Uprimny, R. y Saffon, M. (2006). Justicia transicional y justicia restaurativa: tensiones y comple- mentariedades. En Uprimny, R., editor, ¿Justicia transicional sin transición?: verdad, justicia y reparación para Colombia. Centro de Estudios de Derecho, Justicia y Sociedad.

Verloo, M. y van Lamoen, I. (2002). Policy frames and implementation problems: the case of gender mainstreaming. 